\title{
MemoRob: Studying Robots Distractor Effects
} \author{
Tijus $^{1}$ \\ ${ }^{1}$ CHArt Laboratory, Paris 8 University, France \\ ${ }^{2}$ Lab-STICC Laboratory, South Brittany University, France \\ ${ }^{3}$ ETHOS Laboratory, Rennes I University, France \\ ${ }^{4}$ INSEP, France
}

Céline Jost ${ }^{1}$, Brigitte Le Pévédic ${ }^{2 *}$, Marine Grandgeorge ${ }^{3}$, Marie Le Menn ${ }^{4}$, Farah Arab ${ }^{1}$, M’ballo Seck $^{1}$, Charles

Corresponding Author Email: brigitte.le-pevedic@univ-ubs.fr

https://doi.org/10.18280/mmc_c.811-410

Received: 13 August 2020

Accepted: 1 November 2020

\section{Keywords:}

human-robot interaction, learning, cognitive stimulation, attention, distraction

\begin{abstract}
MemoRob is a model about how to optimize the use of robots for learning. It is based on a list of each possible robotic source of distraction associated with its relevant effects according to its nature and to the target learning mode. While collecting the sources of distraction that the robotics literature provides, the instantiation method of pairing each source and each learning mode with the nature of the distraction as well as its distracting effects allows to consider how to remedy these effects of robotic distraction effects although still having the robotic input as a learning medium. In this article, we provide the motivations that led to the need for the MemoRob model, the list of sources and effects generated by the Human-Robot interaction that may interfere with learning situations, the learning modes described according to their processes and mechanisms and, finally, a set of predictions on whether a given robotic learning situations might promote attention or distraction.
\end{abstract}

\section{INTRODUCTION}

Robots are more and more daily life smart objects. While they were first industrially used to perform difficult and repetitive tasks [1], since the $90 \mathrm{~s}$, they are companion and service robots populating our environment: receptionists at airports, supermarket lobbies, large companies, museums, train stations. They also put teachers in touch with their sick pupils (tele-presence) or help pupils in difficulty. They assist nursing staff in retirement homes, rehabilitation centers and hospitals. They are also used for entertainment or to provide various types of aids [2]. That currently leads researchers to concentrate on ethical problems which have to be solved: "the potential reduction in the amount of human contact due to an increase in the feelings of objectification and loss of control, loss of privacy; loss of personal liberty but also deception and infantilisation" [3]. They deal the circumstances in which elderly people should be allowed to control robots, as well as questions such as: What is the role of robots? How do they must behave to integrate in the society without damaging people? What is their psychological impact on people? How to protect private life?

Robots daily presence could provide real benefits to the elderly. For example, they can decrease loneliness per se, but also by keeping social links with families and caregivers, thanks to video calls-, and reminding meetings. They can improve safety by clocking time to take medicine, calling emergencies in case of danger $[4,5]$. They also could help people with Autism spectrum disorder (ASD) by favoring social contacts and by guiding them in their learning and socialization [6-8]. In addition, they can also propose cognitive stimulation exercises or being teachers assistants $[9$, $10]$.

But, is it judicious to use robots for doing tasks that impact cognition? Indeed, in a situation in which a human is learning or training cognitive functions, s/he has to be fully attentive, concentrated and active. The mind activity is then regulated by attention, which is a sine qua non condition for learning [11] [12]. This focalized attention of someone interacting with a robot is precisely the topic at the root of our work, taking into account that motions, noise and even the robot shape are known to attract or distract attention [13-15].

So, by their nature, robots capture humans' attention and thus, they could distract them from their cognitive tasks although numerous studies show that robots can increase humans' engagement and motivation [16, 17] as well as cognitive performances [18]. This is why the MemoRob project and model aim at mastering the robots' distractor effects to allow humans efficiency when performing learning exercises, without loss of attention being detrimental to cognitive work.

In the followings, this paper is about this complex problematic. Section 2 gives a definition of attention and introduces a discussion about researches to be conducted to investigate the balance between attention and distraction when the robot intervenes during learning tasks. According to the state of the art of the literature, section 3 introduces the more studied effects of Human-Robot Interaction (HRI). Section 4 presents the cognitive processes involved in learning tasks and the effects of robot induced distraction that could impede knowledge and know-how acquisition. Finally, section 5 is about concluding remarks. 


\section{ATTENTION AND DISTRACTION}

\subsection{What's about attention?}

In a general way, attention is defined as a control mechanism of behavioral and cognitive functioning, which contributes to information perception and selection, thus to information treatment. More precisely, it could easily be argued that the allocation of attention can be voluntary or involuntary [19]:

-Voluntary attention which is endogenous, controlled, and top-down guided by a task execution

-Involuntary attention which is exogenous, automatic, attracted by an element in the environment on which one was not focused on before.

\subsection{What's about distraction?}

In opposite to attention, distraction is the cognitive attraction to inadequate objects of the task at hand (distractors) and the paying of attention of these are then impairing attention to the adequate objects of the current task. Thus, in a classroom, if a pupil interest for something else the teacher tells or does, one could say that this pupil is subjected to distraction because what is told or done in the classroom should be object of attraction. This observation that the pupil is not attentive and that $\mathrm{s} / \mathrm{he}$ is distracted is a normative observation that is made from a point of view external to the pupil. This external point of view, that can be those of the teacher, is not those of the pupil who can be very attentive to what "distracts" her/him. To study, understand and model learning, one should opt for an approach based on the learner's point of view. Thus, the learner model would consider that this one focuses her/his attention on what s/he is doing that is, thus, source of attraction and being consequently a source of distraction from other activities.

\subsection{Control and measure of attention-distraction}

An important number of research is dedicated to humans' engagement in Human-Robot Interaction. Studies from several disciplines show that the more complex is the interaction, the more lengthily it is likely to capture attention [20]. For example, if a robot uses several modalities to interact with a human, it will attract human's attention more easily [21]. For instance, adding an auditory feedback to a visual task allows decreasing the level of distraction and, thus, decreasing the number of mistakes done par the user [22]. Another way to favor engagement consist through eye contact for capturing more lengthily the participant attention [23, 24]. Finally, when the user neglects the robot for another task, one among possible strategies to redirect human attention consists slightly trying to distract the user from her/his current task with distractors/attractors [20].

However, in order to get a robot that favors users' attention, one needs to evaluate her/his level of attention. A literature review allows identifying three indicators of attention/distraction: gaze analysis, human behavioral analysis, and neuronal activations analysis with EEG sensors (see below). Several studies establish a link between gaze and level of attention [25, 26]; gaze being an important social component and a reliable indicator of the level of interaction between two individuals [27]. In addition, recent studies find a correlation between EEG sensors data and pupil dilatation, which would indicate mental load and, therefore, the level of attention and engagement in the current task [28]. Eyes behavior would be a very good predictor of the level of user's attention.

Researchers also try to measure attention with different modalities. For example, in addition to collect gaze direction, some studies are based on reaction time [15], facial expression, user's physical distance with the robot [29, 30], general behavior comprising verbal and non-verbal behavior [31], posture, as well as ongoing activities [28].

\section{EFFECTS OF HUMAN-ROBOT INTERACTIONS}

Interaction between human and robot (HRI) is a complex process that involves human dimensions, robotic dimensions and their interactions. This section lists the most known effects in Human-Robot Interaction (HRI) according to four dimensions: appearance, history, communication, adaptation.

\subsection{Appearance}

\subsubsection{Anthropomorphism}

Anthropomorphism is defined by Duffy as "the tendency to attribute human characteristics to inanimate objects, animals and other things in order to help us rationalize their actions" [32]. Everything a robot is doing is interpreted by humans and has consequences for how humans perceive the robot. Many studies are about conditions under which humans anthropomorphize robots by emphasizing similarities between robots and humans $[33,34]$.

\subsubsection{Incarnation}

Among anthropomorphism properties, physical similarity appears to be related to human acceptance of the robot [16] [35]. Among the major results, the literature shows that physical embodiment of an agent offers a more immersive user experience, improves game feedback and makes social interaction more believable [8]. Incarnation in a physical element even has a positive influence on the interaction with a person in telepresence by increasing its social impact, familiarity and directivity [36].

Recent studies are about the notion of re-embodiment where we consider the intelligence of a same robot via several incarnations [37], just as it is done when talking successively with a single person by phone, in real life, by video, etc.

Finally, the valley of the strange [38] defines a threshold beyond which the appearance of the robot causes its rejection. The more the robot looks like human but without fully controlling the associated behavior, the more the level of discomfort of the user in HRI increases.

\subsubsection{Effect of robot gender}

Studies show that gendering the robot has an impact on the interaction. For example, Zhumabekova et al. have shown that a majority of children prefer to play with a robot of the same type as them [39]. Although not representative, this result is indicating that we must ask ourselves the question of the robot's gender. 


\subsection{History}

\subsubsection{Novelty effect}

Faced with a technological novelty, humans express a behavior that is not representative of their long-term feelings. Thus, in order to study the set of long-term interactions between humans and robots, one needs to wait for this novelty effect to pass [40].

\subsubsection{Habituation effect}

Once novelty effect wears off, a habituation effect sets in which can cause disinterest for such an innovative technology. This is why humans may change their opinion of the robot over time [41]. The challenge then is to find strategies to engage humans in the interaction.

\subsubsection{Age of human}

The age of the participant in HRI is influencing on human expectations about the robot and therefore on the interaction [42]. As a matter of fact, many studies differentiate child-robot interaction [43] and senior-robot interaction [3].

\subsection{Communication}

\subsubsection{Effect of physical presence}

Unlike a virtual character, a physical robot, because of its sharing of the physical environment with humans, is perceived as "more engaging, more pleasant to interact with, more informative and credible" [16], it inspires more easily trust and respect [44], and it provides more immersive user experiences, better feedback and more credible social interaction [17].

The presence of a physical robot is also increasing positive emotions and will not be increasing the stress of a same situation experienced without a robot [30]. It is also shown that humans generally have more empathy for physical than virtual robots [45].

Finally, it seems that the physical presence allows the robot to have a natural role of coaching [46] and, thus, improves the performance of the coaches. For instance, participants showed a higher retention rate of movements to reproduce when the learning was guided by a robot [47].

\subsubsection{Audience effect}

The presence of an observer influences the performance of humans. This is the audience effect, which is also observed when the observer is a humanoid robot $[47,48]$ or a group of several robots [49] (which seem to promote better human immersion in the HRI). The appearance of the robot also plays an important role here, since the audience experience is negatively appreciated with a group of non-humanoid robots [49].

Notice however that the audience effect seems to apply only in certain cases, for example in the case of the Stroop task that requires attentional control for successful performance [50].

\subsubsection{Gaze effect}

Studies show that humans seek eye contact with robots just like they do with other humans. This has important consequences for the interaction, for example, humans are more engaged in communication if two robots are looking at them [49].

\subsubsection{Behavior effect}

Many studies have paid attention at the effects of the behavior of the robot. For example, studies show that an emotional robot improves human perception and joy [9, 51]. It should also be noticed that endowing the robot with social behavior seems to increase human learning performance [52], while inappropriate behavior causes discomfort for the user [30].

There is also a positive effect on long-term memorization of new words and an overall higher level of engagement during learning activities when robotic gestures are used [53]. In addition, previous studies have shown the importance of congruence between gesture and speech on the robot credibility [54].

\subsection{Adaptation}

\subsubsection{Expectations}

Even before the taking place of interaction with a robot, humans have expectations that are affecting directly how they perceive the interaction. For example, the user expects a robotic cat to behave as a real cat, at the risk of rejecting the robot. However, the level of expectation is quite different if the robot is a humanoid, a familiar animaloid, an unfamiliar animaloid, or an imaginary one (from the most expected to the least expected) [4].

In addition, with the emergence of social robotics, robots are judged and scored through human subjective emotional measures (joy, well-being), and no longer only with objective measures (speed, precision), which places humans at the heart of interaction and evaluation [4].

\subsubsection{Anticipation of humans}

Humans prefer interacting with a robot whose behavior they can predict. Such an ability to anticipate interaction increases anthropomorphism towards the robot and its acceptance [33].

\subsubsection{Interaction effects}

All of these effects impact on each other.

For example, Park et al. have shown that the behavior of the robot and its appearance are both important, but that if they are contradictory then it is the behavior of the robot that predominates over its appearance in human perception [55]. A good illustration is the study which shows that the robot is judged to be smarter and more appreciable if it makes subtle jokes [56].

Literature also shows that the robot's appearance and its context have an impact on robot acceptance. For example, a "product-oriented" robot increases consumers' purchasing intention more than a "human-oriented" robot. Thus, expectations and context are playing an important role in the acceptance of the robot [57]. More generally, in a social context, humans prefer to interact with a humanoid robot, while in a task context, humans prefer to interact with an industrial robot [58]. 


\section{COGNITION AND LEARNING}

As seen in section I, robots are often used in learning situations according to various paradigms [6-10]. They can be used of course for learning robotics, for learning with robotics with a robot that can be used for demonstration, or for teaching with robotics with a robot that can teach [59]. A robot can also be used to motivate the child by pretending being in a student position and asking the child to teach it ; which forces the child to master what $\mathrm{s} /$ he must transmit to the robot and to have a meta-pedagogical reflection by asking her/himself how to explain the robot and to make it understand and learn [60]. Finally, the robot is also used to study learning processes by being the experimenter that can evaluate the child knowledge and know-how. In that case, the literature reports are that the child behaves more rationally than facing a human being to whom $\mathrm{s} /$ he attributes intentions and ways of doing according to the situations and contexts [61] and according to its commitment to the task at hand [62].

While robots can be a learning aid in all of these situations, they can also be a source of distraction. Based on what we know about learning processes and mechanisms, let's identify how robots might contribute to the attractiveness upon which distraction is based.

Learning is internalization of knowledge that is first external to the learner. There are ways of learning know-how and knowledge [63] and different learning mechanisms. All modes of learning aim at an internal representation of knowledge of the external world [64]. This representation corresponds to networks of categories which are mainly hierarchized according to one type of relationship for knowhow (troponymy: a manner of doing something) and two other types for knowledge about things in the world (taxonomy: kind of and meronymy: part of) [65]. To these categories are attached properties, including know-how and action, which allow the creation of so-called "natural" categories and especially the use of these categories to detect, recognize and then identify a new object, a new relationship, a new way of doing things $[66,67]$. Finally, there is a primacy of action over knowledge conceptualization: It is through action that categories of the objects and concepts are built [68] and it is through function (what's for) and execution (how to) that the category of useful objects of everyday life are defined and refined $[69,70]$.

The learning of know-how can be done by many ways: there are learning by imitation, learning by doing, learning by problem solving, procedural learning of how to perform tasks $[71,72]$. As for the learning of knowledge: there are verbal learning, learning through education and teaching [73], learning through reading and learning through writing verbal production [74].

Among the learning mechanisms, there are learning by analogy [75] and by categorization [76], and by accretion, tuning and restructuring [77].

For all of these learning modes and mechanisms, the robot can play a positive role by controlling and reducing the distracting effects of diverse factors that could have an impact (see section III), or being source of distraction affecting any of these learning modes and mechanisms. The science of robotics interaction is be used to predict the negative or positive effects of using robots for learning according to learning modes and robot intervention mode that promotes attention or distraction.

\section{CONCLUSION AND PERSPECTIVES}

The next step of our research is to define a robust MemoRob model. Our aim is to base this Human-Robot Interaction (HRI) Model on the measurement processing of human attention in order to determine the conditions that make it possible to maintain or increase the user's engagement and, to do so by provide an appropriate response. For instance, if a user is losing her/his attention, being disengaged from the HRI because being engaged in another current task, - or trying to -, one way is to distract her/him from this alternative candidate task in order to re-attract her/his attention to the IHR, taking advantage of the auspicious times.

Our goal is to set up a series of experiments to test the model's predictions in situations that promote either attention or distraction; this in order to be able to offer optimal learning situations.

We are currently working on the design and development of an experimentation and evaluation platform devised to compare a real robot, the same robot in its virtual version (its avatar) and with a tablet for differentiating among these HRI modes. This platform will allow experiments to be carried out by comparing a control situation, a situation with a physical robot and a situation with a virtual robot. The next step will be to address effects that can be controlled, for example the novelty effect and the habituation effect in the context of dedicated scenarios. Finally, a last step will be defining a set of experiments to measure the impact of the robot's effects, as well as the MemoRob remediation on human learning.

\section{ACKNOWLEDGMENTS}

We would like to thank Paris 8 University which is funding the first stage of the project. We also thank Aurélie Bucaille and Grégory Legouverneur who participated in the project in its early steps in order to contribute their knowledge on memory. And finally, we would like to thank Isabelle Xue, Ali Mahfoufi and Mohamed Benomari who made the first IT developments.

\section{REFERENCES}

[1] Shibata, T. (2004). An overview of human interactive robots for psychological enrichment. Proceedings of the IEEE, 92(11): https://doi.org/1109/JPROC.2004.835383

[2] Baraka, K., Alves-Oliveira, P., Ribeiro, T. (2020). An extended framework for characterizing social robots. Human-Robot Interaction, 21-64. https://doi.org/10.1007/978-3-030-42307-0_2

[3] Sharkey, A., Sharkey, N. (2012). Granny and the robots: ethical issues in robot care for the elderly. Ethics and Information Technology, 14(1): 27-40. https://doi.org/10.1007/s10676-010-9234-6

[4] Wada, K., Shibata, T., Asada, T., Musha, T. (2007). Robot therapy for prevention of dementia at home. Journal of Robotics and Mechatronics, 19(6): 691-697.

[5] Chetouani, M., Wu, Y. H., Jost, C., Le Pevedic, B., Fassert, C., Cristancho-Lacroix, V., Rigaud, A.S. (2010). Cognitive services for elderly people: the ROBADOM project. ECCE workshop: Robots that care, Delf, Netherlands. 
[6] Robins, B., Dautenhahn, K., Te Boekhorst, R., Billard, A. (2005). Robotic assistants in therapy and education of children with autism: can a small humanoid robot help encourage social interaction skills?. Universal access in the information society, 4(2): 105-120. https://doi.org/10.1007/s10209-005-0116-3

[7] Ricks, D.J., Colton, M.B. (2010). Trends and considerations in robot-assisted autism therapy. 2010 IEEE International Conference on Robotics and Automation, Anchorage, AK, pp. 4354-4359. https://doi.org/10.1109/ROBOT.2010.5509327

[8] Dautenhahn, K., Werry, I. (2002). A quantitative technique for analysing robot-human interactions. IEEE/RSJ International Conference on Intelligent Robots and Systems, Lausanne, Switzerland, 2002, pp. 1132-1138 vol.2, doi: 10.1109/IRDS.2002.1043883

[9] Tapus, A., Vieru, A.M. (2013). Robot cognitive stimulation for the elderly. International WorkConference on the Interplay Between Natural and Artificial Computation, 94-102 https://doi.org/10.1007/978-3-642-38637-4_10

[10] Edwards, A., Edwards, C., Spence, P.R., Harris, C., Gambino, A. (2016). Robots in the classroom: Differences in students' perceptions of credibility and learning between "teacher as robot" and "robot as teacher". Computers in Human Behavior, 65: 627-634. https://doi.org/10.1016/j.chb.2016.06.005

[11] Posner, M.I., Dehaene, S. (1994). Attentional networks. Trends in Neurosciences, 17(2): 75-79. https://doi.org/10.1016/0166-2236(94)90078-7

[12] Naccache, L., Blandin, E., Dehaene, S. (2002). Unconscious masked priming depends on temporal attention. Psychological science, 13(5): 416-424. https://doi.org/10.1111/1467-9280.00474

[13] Abrams, R.A., Christ, S.E. (2003). Motion onset captures attention. Psychological Science, 14(5): 427-432. https://doi.org/10.1111/1467-9280.01458

[14] Franconeri, S.L., Simons, D.J. (2003). Moving and looming stimuli capture attention. Perception \& psychophysics, 65(7): 999-1010 https://doi.org/10.3758/BF03194829

[15] Li, X.A., Florendo, M., Miller, E.L., Ishiguro, H., Saygin, P. A. (2015, March). Robot form and motion influences social attention. 2015 10th ACM/IEEE International Conference on Human-Robot Interaction (HRI), Portland, OR, pp. 43-50.

[16] Kidd, C.D., Breazeal, C. (2004). Effect of a robot on user perceptions. 2004 IEEE/RSJ International Conference on Intelligent Robots and Systems (IROS) (IEEE Cat. No.04CH37566), Sendai, pp. 3559-3564. https://doi.org/10.1109/IROS.2004.1389967.

[17] Pereira, A., Martinho, C., Leite, I., Paiva, A. (2008). iCat, the chess player: the influence of embodiment in the enjoyment of a game. Proceedings of the 7th International Joint Conference on Autonomous Agents and Multiagent Systems, 3: 1253-1256.

[18] Belpaeme, T., Kennedy, J., Ramachandran, A., Scassellati, B., Tanaka, F. (2018). Social robots for education: A review. Science robotics, 3(21): eaat5954. https://doi.org/10.1126/scirobotics.aat5954

[19] Wundt, W. (1897). Grundriss der psychologie, von Wilhelm Wundt. W. Engelmann.

[20] Cools, R., Simeone, A.L. (2019). Investigating the effect of distractor interactivity for redirected walking in virtual reality. SUI 2019: ACM Conference on Spatial User Interaction, pp. 1-5. https://doi.org/10.1145/3357251.3357580

[21] Saad, E., Neerincx, M.A., Hindriks, K.V. (2019). Welcoming robot behaviors for drawing attention. 2019 14th ACM/IEEE International Conference on HumanRobot Interaction (HRI), Daegu, Korea (South), pp. 636637. https://doi.org/10.1109/HRI.2019.8673283

[22] Secoli, R., Rosati, G., Reinkensmeyer, D.J. (2009). Using sound feedback to counteract visual distractor during robot-assisted movement training. 2009 IEEE International Workshop on Haptic Audio visual Environments and Games, Lecco, pp. 135-140. https://doi.org/10.1109/HAVE.2009.5356119

[23] Das, D., Hoque, M.M., Kobayashi, Y., Kuno, Y. (2013). Attention control system considering the target person's attention level. 2013 8th ACM/IEEE International Conference on Human-Robot Interaction (HRI), Tokyo, 2013, pp. 111-112. https://doi.org/10.1109/HRI.2013.6483526

[24] Hoque, M.M., Onuki, T., Das, D., Kobayashi, Y., Kuno, Y. (2012). Attracting and controlling human attention through robot's behaviors suited to the situation. Proceedings of the seventh annual ACM/IEEE International Conference on Human-Robot Interaction pp. 149-150. https://doi.org/10.1145/2157689.2157729

[25] Birmingham, E., Kingstone, A. (2009). A New Look at Past, Present, and Future Investigations. Annals of the New York Academy of Sciences, 1156: 118-140.

[26] Ames, C., Fletcher-Watson, S. (2010). A review of methods in the study of attention in autism. Developmental Review, 30(1): 52-73. https://doi.org/10.1016/j.dr.2009.12.003

[27] Emery, N. J. (2000). The eyes have it: the neuroethology, function and evolution of social gaze. Neuroscience \& Biobehavioral Reviews, 24(6): 581-604. https://doi.org/10.1016/S0149-7634(00)00025-7

[28] Anzalone, S.M., Boucenna, S., Ivaldi, S., Chetouani, M. (2015). Evaluating the engagement with social robots. International Journal of Social Robotics, 7(4): 465-478. https://doi.org/10.1007/s12369-015-0298-7

[29] Attamimi, M., Miyata, M., Yamada, T., Omori, T., Hida, R. (2016). Attention estimation for child-robot interaction. Proceedings of the Fourth International Conference on Human Agent Interaction, pp. 267-271. https://doi.org/10.1145/2974804.2980510

[30] Jost, C., Grandgeorge, M., Le Pévédic, B., Duhaut, D. (2014). Robot or tablet: Users' behaviors on a memory game. The 23rd IEEE International Symposium on Robot and Human Interactive Communication, Edinburgh, pp. 1050-1055. https://doi.org/10.1109/ROMAN.2014.6926391

[31] Rochais, C. (2018). Attention-getting behaviors. Encyclopedia of Animal Cognition and Behavior, Springer International Publishing, pp. 1-6. https://doi.org/10.1007/978-3-319-47829-6_248-1

[32] Duffy, B.R. (2003). Anthropomorphism and the social robot. Robotics and Autonomous Systems, 42(3-4): 177190. https://doi.org/10.1016/S0921-8890(02)00374-3

[33] Eyssel, F., Kuchenbrandt, D., Bobinger, S. (2011). Effects of anticipated human-robot interaction and predictability of robot behavior on perceptions of anthropomorphism. Proceedings of the 6th international conference on Human-robot interaction, pp. 61-68. 
https://doi.org/10.1145/1957656.1957673

[34] Zlotowski, J., Bartneck, C. (2013, March). The inversion effect in HRI: Are robots perceived more like humans or objects?. 2013 8th ACM/IEEE International Conference on Human-Robot Interaction (HRI), Tokyo, pp. 365-372. https://doi.org/10.1109/HRI.2013.6483611

[35] Jost, C., André, V., Le Pévédic, B., Lemasson, A., Hausberger, M., Duhaut, D. (2012). Ethological evaluation of Human-Robot Interaction: are children more efficient and motivated with computer, virtual agent or robots? 2012 IEEE International Conference on Robotics and Biomimetics (ROBIO), Guangzhou, pp. 1368-1373.

https://doi.org/10.1109/ROBIO.2012.6491159

[36] Kawaguchi, I., Kodama, Y., Kuzuoka, H., Otsuki, M., Suzuki, Y. (2016). Effect of embodiment presentation by humanoid robot on social telepresence. Proceedings of the Fourth International Conference on Human Agent Interaction, pp.

$253-256$ https://doi.org/10.1145/2974804.2980498

[37] Reig, S., Forlizzi, J., Steinfeld, A. (2019). Leveraging robot embodiment to facilitate trust and smoothness. 2019 14th ACM/IEEE International Conference on Human-Robot Interaction (HRI), Daegu, Korea (South), pp. 742-744. https://doi.org/10.1109/HRI.2019.8673226

[38] Mori, M. (1970). The uncanny valley, Energy, 7(4): 33$35,1970$.

[39] Zhumabekova, K., Ismailova, A., Kushkinbayev, D., Sandygulova, A. (2018). Exploring the Effects of Robot Gender on Child-Robot Interaction. In Companion of the 2018 ACM/IEEE International Conference on HumanRobot Interaction, pp. 287-288. https://doi.org/10.1145/3173386.3177044

[40] Dautenhahn, K. (2004). Robots we like to live with?!-a developmental perspective on a personalized, life-long robot companion. In RO-MAN 2004. RO-MAN 2004. 13th IEEE International Workshop on Robot and Human Interactive Communication (IEEE Catalog No.04TH8759), Kurashiki, Okayama, Japan, pp. 17-22. https://doi.org/10.1109/ROMAN.2004.1374720

[41] Koay, K.L., Syrdal, D.S., Walters, M.L., Dautenhahn, K. (2007). Living with robots: Investigating the habituation effect in participants' preferences during a longitudinal human-robot interaction study. RO-MAN 2007 - The 16th IEEE International Symposium on Robot and Human Interactive Communication, Jeju, pp. 564-569. https://doi.org/10.1109/ROMAN.2007.4415149

[42] Baxter, P., De Jong, C., Aarts, R., de Haas, M., Vogt, P. (2017). The effect of age on engagement in preschoolers' child-robot interactions. Proceedings of the Companion of the 2017 ACM/IEEE International Conference on Human-Robot Interaction, pp. 81-82. https://doi.org/10.1145/3029798.3038391

[43] Belpaeme, T., Baxter, P., De Greeff, J., Kennedy, J., Read, R., Looije, R., Zelati, M.C. (2013). Child-robot interaction: Perspectives and challenges. International Conference on Social Robotics, pp. 452-459. https://doi.org/10.1007/978-3-319-02675-6_45

[44] Bainbridge, W.A., Hart, J., Kim, E.S., Scassellati, B. (2008). The effect of presence on human-robot interaction. RO-MAN 2008 - The 17th IEEE International Symposium on Robot and Human Interactive Communication, Munich, pp. 701-706. https://doi.org/10.1109/ROMAN.2008.4600749
[45] Seo, S.H., Geiskkovitch, D., Nakane, M., King, C., Young, J.E. (2015). Poor thing! Would you feel sorry for a simulated robot? A comparison of empathy toward a physical and a simulated robot. 2015 10th ACM/IEEE International Conference on Human-Robot Interaction (HRI), Portland, OR, pp. 125-132.

[46] Jost, C., Grandgeorge, M., Le Pévédic, B., Duhaut, D. (2013, October). Are robots our future coaches?. IEEE ISR 2013, Seoul, pp. 1-6. https://doi.org/10.1109/ISR.2013.6695626

[47] Kosaka, A., Katakura, T., Toyama, S., Ikeda, F. (2018, March). Evaluation of Posture Memory Retentivity using Coached Humanoid Robot. Companion of the 2018 ACM/IEEE International Conference on Human-Robot Interaction,

pp. 159-160. https://doi.org/10.1145/3173386.3176974

[48] Fraune, M.R., Sherrin, S., Sabanović, S., Smith, E.R. (2015). Rabble of robots effects: Number and type of robots modulates attitudes, emotions, and stereotypes. Proceedings of the Tenth Annual ACM/IEEE International Conference on Human-Robot Interaction, pp. 109-116. https://doi.org/10.1145/2696454.2696483

[49] Ichijo, T., Munekata, N., Hiraki, K., Ono, T. (2014). Entrainment effect caused by joint attention of two robots. Proceedings of the 2014 ACM/IEEE international conference on Human-robot interaction, pp. 178-179. https://doi.org/10.1145/2559636.2559851

[50] Spatola, N., Belletier, C., Chausse, P., Augustinova, M., Normand, A., Barra, V., Huguet, P. (2019). Improved cognitive control in presence of anthropomorphized robots. International Journal of Social Robotics, 11(3): 463-476. https://doi.org/10.1007/s12369-018-00511-w

[51] Leite, I., Pereira, A., Martinho, C., Paiva, A. (2008). Are emotional robots more fun to play with?. RO-MAN 2008 - The 17th IEEE International Symposium on Robot and Human Interactive Communication, Munich, pp. 77-82. https://doi.org/10.1109/ROMAN.2008.4600646

[52] Ushida, H. (2010). Effect of social robot's behavior in collaborative learning. 2010 5th ACM/IEEE International Conference on Human-Robot Interaction (HRI), Osaka, pp. 195-196. https://doi.org/10.1109/HRI.2010.5453199

[53] de Wit, J., Schodde, T., Willemsen, B., Bergmann, K., de Haas, M., Kopp, S., Vogt, P. (2018). The effect of a robot's gestures and adaptive tutoring on children's acquisition of second language vocabularies. Proceedings of the 2018 ACM/IEEE International Conference on Human-Robot Interaction, pp. 50-58. https://doi.org/10.1145/3171221.3171277

[54] Jost, C., Pévédic, B. L., Duhaut, D. (2012). Study of the importance of adequacy to robot verbal and non verbal communication in human-robot interaction. arXiv preprint arXiv:1206.3002.

[55] Park, E., Kong, H., Lim, H.T., Lee, J., You, S., del Pobil, A.P. (2011). The effect of robot's behavior vs. appearance on communication with humans. Proceedings of the 6th International Conference on Human-Robot Interaction, pp. 219-220. https://doi.org/10.1145/1957656.1957740

[56] Menne, I.M., Lange, B.P., Unz, D.C. (2018). Мy humorous robot: effects of a robot telling jokes on perceived intelligence and liking. Companion of the 2018 ACM/IEEE International Conference on Human-Robot Interaction,

pp.

193-194. 
https://doi.org/10.1145/3173386.3177015

[57] Kwak, S.S., San Kim, J., Choi, J.J. (2014). Can robots be sold? The effects of robot designs on the consumers' acceptance of robots. 2014 9th ACM/IEEE International Conference on Human-Robot Interaction (HRI), Bielefeld, 2014, pp. 220-221.

[58] Choi, J.J., Kwak, S.S. (2015). The effect of robot appearance types and task types on Service evaluation of a robot. Proceedings of the Tenth Annual ACM/IEEE International Conference on Human-Robot Interaction Extended Abstracts, pp. 223-224. https://doi.org/10.1145/2701973.2702735

[59] Gaudiello, I., Zibetti, E. (2016). Learning robotics, with robotics, by robotics: Educational robotics. John Wiley $\&$ Sons.

[60] Masson, O., Ruggieri, F. (2019). Robotique éducative pour la découverte, la réflexion et le raisonnement. Enfance, (3): 333-344.

[61] Masson, O., Baratgin, J., Jamet, F., Ruggieri, F., Filatova, D. (2016). Use a robot to serve experimental psychology: Some examples of methods with children and adults. 2016 International Conference on Information and Digital Technologies (IDT), Rzeszow, pp. 190-197. https://doi.org/10.1109/DT.2016.7557172

[62] Ivaldi, S., Lefort, S., Peters, J., Chetouani, M., Provasi, J., Zibetti, E. (2017). Towards engagement models that consider individual factors in HRI: On the relation of extroversion and negative attitude towards robots to gaze and speech during a human-robot assembly task. International Journal of Social Robotics, 9(1): 63-86. https://doi.org/10.1007/s12369-016-0357-8

[63] Tijus, C. (2013). Know-How modelling for e-Learning. Proceedings - 2013 RIVF International Conference on Computing and Communication Technologies: Research, Innovation, and Vision for Future, RIVF 2013, pp. 186191.

[64] Chang, C. Y., Tijus, C., Zibetti, E. (2015). Les apprentissages à l'heure des technologies cognitives numériques. Administration Education, (2): 91-98.

[65] Le Ny, J.F. (2002). Predicates, events and situations: a cross-cognitive analysis and reconsideration of some basic notions of discourse comprehension. Trends in
Cognitive Psychology, pp. 216-236.

[66] Bonnardel, N., Didierjean, A., Marmèche, E. (2003). Analogie et résolution de problèmes. Métaphores Analog., 115-149.

[67] Tijus, C., Brezillon, P., Tijus, C., Brezillon, P. (2006). Problem solving and creativity for decision support systems. Creativity and Innovation in Decision Making and Decision Support, 1: 277-293.

[68] Piaget, J. (2015). The Grasp of Consciousness (Psychology Revivals): Action and Concept in the Young Child. Psychology Press.

[69] Rosch, E., Lloyd, B.B. (1978). Principles of categorization. Cogn. Categ..

[70] Mervis, C.B., Rosch, E. (1981). Categorization of natural objects. Annual review of psychology, 32(1): 89-115. https://doi.org/10.1146/annurev.ps.32.020181.000513

[71] Nadel, J. (2014). How imitation boosts development: In infancy and autism spectrum disorder. OUP Oxford.

[72] Langley, P., Simon, H.A. (1981). The central role of learning in cognition. Cognitive Skills and Their Acquisition, 361-380.

[73] Jamet, F., Masson, O., Jacquet, B., Stilgenbauer, J.L., Baratgin, J. (2018). Learning by teaching with humanoid robot: a new powerful experimental tool to improve children's learning ability. Journal of Robotics. ttps://doi.org/10.1155/2018/4578762

[74] Caillies, S., Denhière, G., Kintsch, W. (2002). The effect of prior knowledge on understanding from text: Evidence from primed recognition. European Journal of Cognitive Psychology, 14(2): 267-286. https://doi.org/10.1080/09541440143000069

[75] Gentner, D., Holyoak, K.J. (1997). Reasoning and learning by analogy: Introduction. American Psychologist, 52(1): 32-32. https://doi.org/10.1037/0003-066X.52.1.32

[76] Barsalou, L. W. (1986). Are there static category representations in long-term memory?. Behavioral and Brain Sciences, 9(4): 651-652. https://doi.org/10.1017/S0140525X00051591

[77] Rumelhart, D.E., Norman, D.A. (1978). Accretion, tuning, and restructuring: Three modes of learning. Semantic factors in cognition, 37-53. 